\title{
Akupunktur zur Behandlung von Schmerzen und Funktionsstörungen bei Kniegelenksarthrose
}

\author{
Gonarthrose ist eine der \\ häufigsten degenerativen \\ Gelenkerkrankungen - \\ Die Behandlung mit Aku- \\ punktur ist hinreichend \\ belegt \\ Franziska Portzky
}

Die Gonarthrose ist eine der häufigsten degenerativen Gelenkerkrankung, deren Inzidenz mit steigendem Alter zunimmt [1]. Bei der primären Form ist die Ätiologie unbekannt, sekundär kann sie nach Verletzung, Entzündungen, bei Systemerkrankungen oder Fehlstellungen entstehen. Die Aktivierung der Arthrose kann akut mit Schmerzen und Erguss einhergehen, im chronischen Verlauf beeinträchtigen den Patienten häufig funktionelle Defizite und Schmerzen zunächst bei Belastung später auch in Ruhe. Die medikamentöse Behandlung, insbesondere mit NSAR, ist oftmals durch Nebenwirkungen beschränkt [2] und intraartikuläre Injektionen bergen das Risiko von Gelenkinfektionen. Als komplementärer Behandlungsansatz bietet sich die Akupunktur an, deren schmerzreduzierende Wirkung in einer Vielzahl von hochwertigen Studien belegt werden konnte [3]. Randomisierte kontrollierte Studien im Rahmen der Modellvorhaben Akupunktur (GERAC, ART) zeigten am bisher größten Patientenkollektiv, dass neben einer Schmerzreduktion auch eine Verbesserung der Gelenkfunktion und eine Reduktion der Schmerzmedikation bei geringer Nebenwirkungsrate möglich ist $[4,5]$. Die Verbesserungen hielten über die

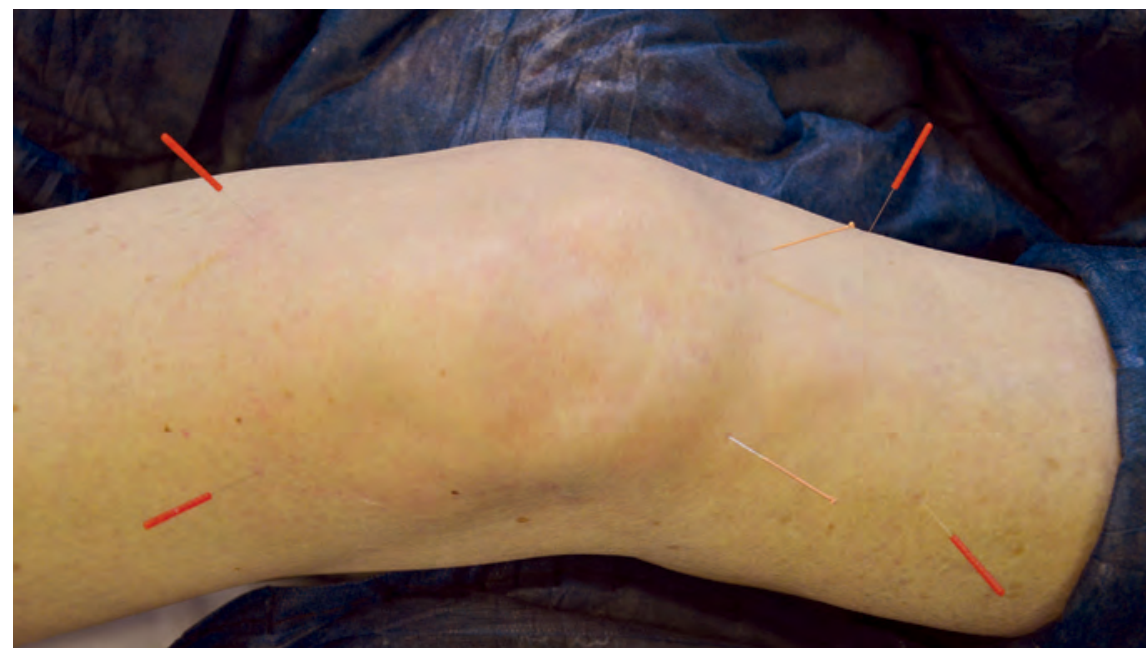

Abb. 1 Lokoregionale Behandlung bei Gonarthrosebeschwerden.

jeweiligen Beobachtungszeiträume, die bis zu 6 Monate umfassten, an. Speziell bei der Gonarthrose zeigte sich auch eine Überlegenheit der punktespezifischen Behandlung gegenüber einer Schein(Sham)-Akupunktur [3]. Mit dem Wirksamkeitsnachweis durch die Modellvorhaben wurde die Akupunktur bei dieser Indikation in die Gebührenordnung der gesetzlichen Krankenkassen aufgenommen.

Die Akupunkturbehandlung richtet sich nach lokoregionalen Gesichtspunkten, nach Leitbahnbezug sowie myofaszial bedingten Schmerzen und Bewegungseinschränkungen. Durch die Syndromdifferenzierung gemäß der Traditionellen Chinesischen Medizin (TCM) kann die Auswahl der Punkte weiter spezifiziert und ergänzende Methoden wie z. B. Moxibustion in die Behandlung mit einbezogen werden. Zusätzlich können Mikrosysteme wie Ohr, Schädel oder Mund

\section{Zusammenfassung}

Die Akupunkturbehandlung bei Gonarthrose ist eine hinreichend belegte Behandlungsmethode, die nebenwirkungsarm ist und neben anderen Behandlungsmethoden angewendet werden kann. Die Akupunkturbehandlung richtet sich nach lokoregionalen Gesichtspunkten, nach Leitbahnbezug sowie myofaszial bedingten Schmerzen und

Bewegungseinschränkungen. Durch die Syndromdifferenzierung gemäß der Traditionellen Chinesischen Medizin (TCM) kann die Auswahl der Punkte weiter spezifiziert und ergänzende Methoden wie z. B. Moxibustion in die Behandlung mit einbezogen werden. Zusätzlich können Mikrosysteme wie Ohr, Schädel oder Mund therapeutisch genutzt werden. 
therapeutisch genutzt werden. Im Folgenden soll dem Praktiker in Anlehnung an u.g. Literaturauswahl [6-11] eine Übersicht über die verschiedenen Aspekte der Behandlung gegeben werden.

\section{Lokoregionale Punkte}

Bei der Lokalbehandlung (Abb.1) ist es wichtig zunächst zu klären, wo der Hauptschmerz besteht bzw. wohin sich der Schmerz erstreckt und die lokoregionalen Punkte der entsprechenden Leitbahn in die Behandlung mit einzubeziehen:

Mediale Knieregion: Milz- (Mi 9,10), Leber(Le 7, 8), oder / und Nierenleitbahn (Ni 10) Laterales Knie: Magen- (Ma 34, 35, 36) und / oder Gallenblasenleitbahn (Gb 33, 34) Ventrales Knie: Magen- und Milzleitbahn (s.o.)

Dorsales Knie: Blasenleitbahn (Bl 39, 40)

Neben der lokalen Wirkung haben viele dieser Punkte zusätzliche Indikationen bezogen auf die Syndrombehandlung nach TCM und können auch für die konstitutionelle Behandlung der Kniebeschwerden verwendet werden.

Zusätzlich zu den lokalen Punkten auf Leitbahnen können Extrapunkte oder druckschmerzhafte, sog. A-Shi-Punkte eingesetzt werden. Häufig (v.a. bei ventralen Schmerzen) werden die „Knieaugen“ (Xiyan) benutzt, worunter man die kombinierte Behandlung vom Extrapunkt UE 4 (Neixiyan) und Ma 35 versteht, medial und lateral der Patellarsehne. Bei Retropatellarschmerzen oder Schmerzen oberhalb der Patella kann Ex-UE2 (Heding) genadelt werden, der in der Mitte des oberen Patellarandes aufzufinden ist.

Bei der lokalen Nadlung ist die Nähe zum Gelenkbinnenraum und den Schleimbeuteln zu beachten. Eine Punktion sollte aufgrund der Infektionsgefahr vermieden werden. Am dorsalen Knie sind zudem A. und V. poplitea oder Baker-Zysten in Betracht zu ziehen, die nicht punktiert werden sollten.

Bei (hoch)akuter bzw. ausgeprägter Symptomatik, wie Rötung, Schwellung, Hautaffektionen, starken Schmerzen, sollte zunächst von einer lokalen Behandlung abgesehen werden und Punkte fern des Lokalgeschehens ausgewählt werden (s.u.). Alternativ kann eine kontralaterale Behandlung am anderen Knie erfolgen, sofern dies nicht betroffen ist.
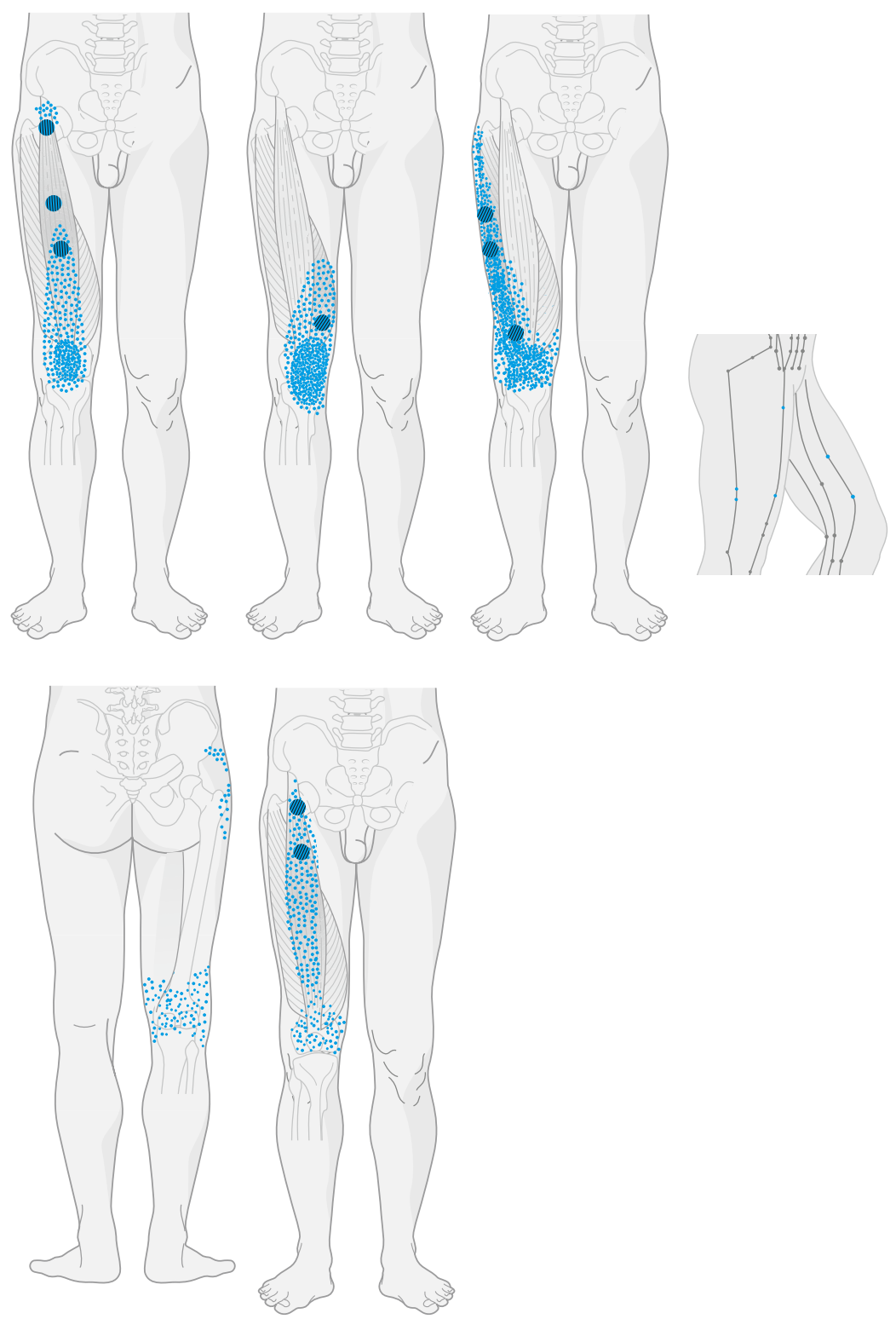

Abb. 2 Ausstrahlung Triggerpunkte. (C [10], mit freundlicher Genehmigung.

\section{Fernpunkte}

Entsprechend der Schmerzlokalisation und der betroffenen Leitbahn(en) werden Fernpunkte in das Behandlungskonzept mit einbezogen. Diese Punkte liegen im Gegensatz zu den lokalen und regionalen Punkten weiter entfernt vom Geschehen und befinden sich v.a. im Bereich von Unterschenkel und Fuß. Wichtige Fernpunkte sind z.B. Gb 40, Ma 41, Mi 5 und Le 3. Für dorsale Schmerzen sind leitbahnbezogene Punkte wie Bl 60 oder 62 angezeigt. Weitere Punkte der Leitbahnen können als Fernpunkte im Rahmen der Syndrombehandlung hinzugenommen werden.
Durch den Achsenbezug finden sich Fernpunkte auch auf den korrespondierenden Leitbahnen der ipsilateralen oberen Extremität. V.a. der Bezug vom Knie- zum Ellenbogengelenk spiegelt sich in dieser Betrachtung wider, wobei häufig verwendete Punkte der Yang-Leitbahnen auch im Schulterbereich liegen.

Yangmingachse (Ma/Di): Di 10 bzw. 15

Shaoyangachse (Gb/3E): 3E 14

Taiyinachse (Mi/Lu): Lu 5 Jueyinachse (Le/Pe): Pe 3

Shaoyinachse (Ni/He): He 3

Taiyangachse (Bl/Dü): Dü 8 oder 10 


\section{Triggerpunktakupunktur}

Die sog. myofaszialen Triggerpunkte ( $\mathrm{mTrP}$ ) stellen einen wichtigen Bestandteil bei der Akupunkturbehandlung von muskulären Dysbalancen dar, die sowohl Ursache als auch Folge von Gonarthrose und ihrer Beschwerden sein können. Vorkenntnisse bezüglich Palpation und Technik sind Voraussetzung für eine gelungene mTrP-Behandlung.

In der körperlichen Untersuchung werden bei seitlichen Kniebeschwerden der M. vastus lateralis und Tractus iliotibialis, sowie der M. vastus medialis, M. adductor magnus und M. gracilis auf druckdolente myofasziale Punkte und Verhärtungen im Sinne von sog. taut bands untersucht. Oft korrelieren diese mit Akupunkturpunkten der Magen / Milz / Gallenblasenleitbahn. Bei einem aktiven TP strahlt der Schmerz in die charakteristische Richtung aus (Abb.2). Bei Schmerzen im Bereich der Patella ist der M. rectus femoris v.a. ursprungsnah und der $\mathrm{M}$. vastus intermedius zu untersuchen. Bei dorsalen Gelenkbeschwerden sind Trigger- punkte im Bereich des M. biceps femoris, M. gastrocnemicus, M. popliteus und M. plantaris zu suchen.

Die gezielte Behandlung erfolgt dann im Rahmen der Triggerpunktakupunktur (Dry needling). Ziel der Nadelung ist das exakte Treffen der mTP und damit das Auslösen einer lokalen Muskelzuckungsreaktion (Local Twitch Response, LTR). Damit werden im besten Fall der Triggerpunkt gelöscht und der Muskel entspannt. Anschließend oder bei ausbleibendem Twitch nach 1-2 Sondierungsversuchen kann die Nadel herausgezogen und das Areal ausgestrichen werden. Die Nadel kann jedoch auch aus dem Muskel zurückgezogen werden und in der Subcutis verbleiben im Sinne einer klassischen Akupunktur. Gefäß-, Nerven- und Gelenkstrukturen sind ebenso wie Entzündungsherde etc. im Nadlungsgebiet abzugrenzen und nicht zu punktieren. Bei Einnahme von blutverdünnenden Medikamenten sollte von einer Triggerpunktakupunktur abgesehen werden wegen der Gefahr größere Hämatome/Einblutungen bis hin zum Kom-

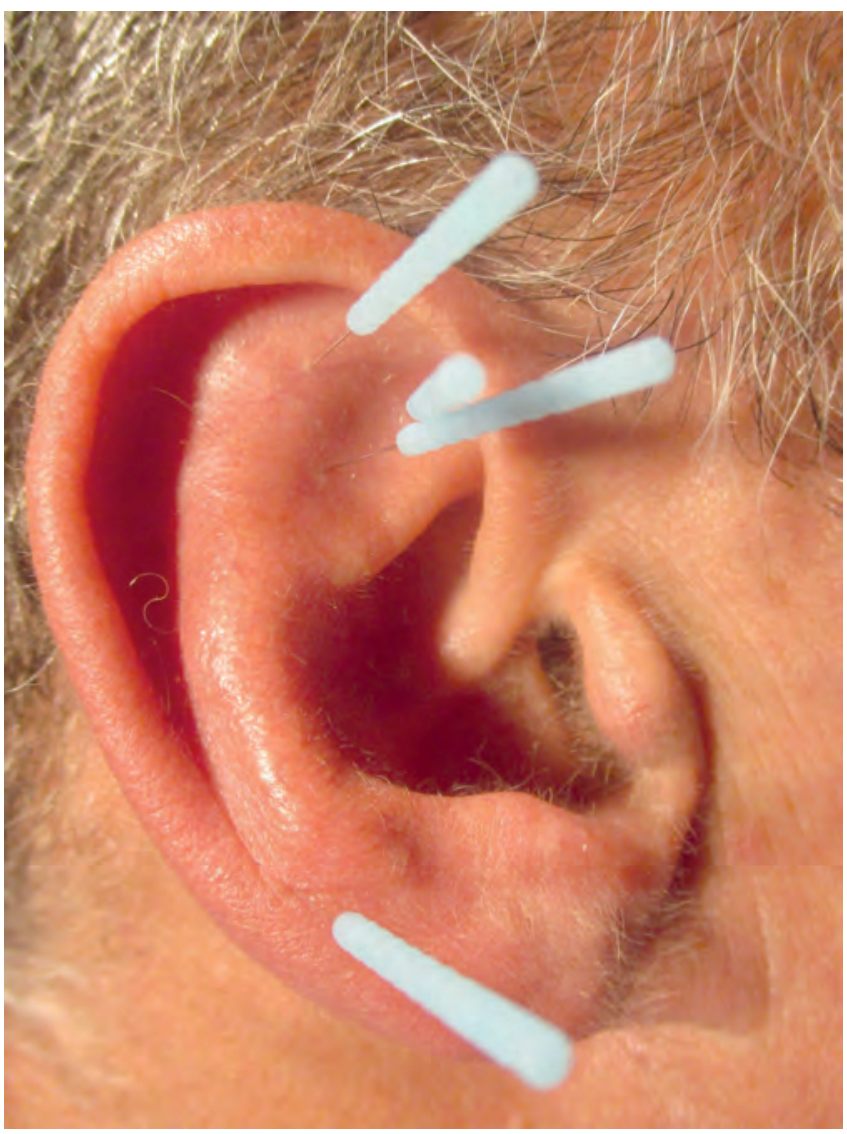

Abb. 3 Ohrakupunktur bei Gonarthrose rechts. partmentsyndrom. Entsprechende Aufklärungen wie bei jeder Akupunktur sind obligat vor der Behandlung, auch sollte der Patient insbesondere bei dieser Behandlung auf einen möglichen über einige Tage anhaltenden Muskelkaterschmerz hingewiesen werden.

In diesem Zusammenhang sei auch auf die Behandlung der außerordentlichen Meridiane hingewiesen. Insbesondere der Yinund der Yang-Qiao-Mai sind für die Regulation des Beinmuskeltonus wichtig, sodass bei einem deutlichen Unterschied zwischen Innen-und Außenmuskulatur der jeweilige Öffnungspunkt (Bl 62 bzw. Ni 6) regulativ genadelt werden kann.

\section{Punkte im Rahmen der Wand- lungsphase bzw. des TCM-Syn- droms}

Beschwerden bei Gonarthrose können im Rahmen von verschiedenen zugrundeliegenden Mustern der TCM gesehen werden. Gerade der Bezug zum Funktionskreis Niere/Blase ist dabei zu beachten. Eine Störung zeigt sich in chronischen Schmerzen und Schwäche der Knie, oft beidseits, und der Wirbelsäule, v.a. der LWS. Miktions-/Sexualdysfunktionen, Erschöpfung, chronische Müdigkeit sprechen ebenfalls für diesen Funktionskreis. Probleme der Hörfähigkeit, Degeneration der Knochen-(Osteoporose) und Hirnsubstanz (Einbußen der kognitiven Fähigkeiten) zeigen gerade im Alter den Bezug zum Funktionskreis Niere. Die Angst als wesentlicher emotionaler Aspekt findet hier sogar umgangssprachlich seinen Ausdruck: Zittern oder Weichwerden der Knie. Zur genaueren Differenzialdiagnose von NierenYin- bzw. -Yang-Mangel können TCM-relevante Kriterien der Leere-Hitze oder -Kälte abgefragt und anhand von Zunge und Puls Hinweise gewonnen werden. Als Behandlungsoption bietet sich in diesen Fällen grundsätzlich die Stärkung des NierenFunktionskreises mittels Shu (Bl 23) und Yuan-Quellpunkt (Ni 3) an, bei Nieren-YangMangel zusätzlich z. B. Du 4, KG 4, KG 6, Ni 7, bei Nieren-Yin-Mangel zusätzlich z.B. Ni 6, Mi 6. Eine tonisierende Nadlung, bei Yang Mangel ggf. unterstützt durch Moxibustion, ist hier angezeigt.

Differenzialdiagnostisch kommt aus Sicht der chinesischen Medizin auch eine lokale Obstruktion der Leitbahnen und Kollateralgefäße in Betracht. Als Ursache kommen Traumata oder pathogene Faktoren wie 
Kälte, Feuchtigkeit, Wind oder Hitze infrage (sog. Bi-Syndrom). Qi-Stagnation und / oder Blut-Stase können die Folge sein. Ein starker lokalisierter Schmerz spricht dabei eher für Blut, ein diffuser, regionaler Schmerz eher für eine Qi-Stagnation. Grundsätzlich bieten sich dabei neben der Lokoregionalbehandlung Punkte an, die generell Qi (z.B. Le 3, Di 4) bzw. Blut (z.B. Bl 17, Mi 10) bewegen.

Beim Bi-Syndrom ist es zudem wichtig, die pathogenen Faktoren, die die Leitbahnen blockieren, zu eliminieren. Im Rahmen der Fernpunktbehandlung können Punkte gewählt werden, die einen Bezug zu dem jeweiligen pathogenen Faktor haben. Symptome wie fixierte Schmerzen, Schwellung des Knies, Schweregefühl, Verschlechterung bei feuchtem Wetter weisen auf eine Ansammlung von Feuchtigkeit hin, die z.B. mittels Mi 5, 6 oder 9 (+Ma 36) ausgeleitet werden kann. Anhaltende, starke, bei Kälte zunehmende Schmerzen weisen auf eine Kälteaffektion hin und können v.a. durch Moxibustion von lokoregionalen Punkten behandelt werden. Ziehende, wandernde, oft bei Wetterwechsel zunehmende Schmerzen können ein Hinweis für ein Wind-Bi-Syndrom sein und können z.B. durch Le 3 mit behandelt werden. Eine Überwärmung des Knies, brennende Schmerzen und Abneigung gegen lokale Wärme weisen auf pathogene Hitze hin, die z.B. mittels den Ying(Quell)Punkten (Ma 44, Mi 2, Le 2) ableitend behandelt werden kann.

\section{MikroAkuPunktSysteme (MAPS)}

Ein weiterer wichtiger Bestandteil der Akupunktur bei Gonarthrose ist die Behandlung über Mikrosysteme. Hierbei soll auf 3 wesentliche Strategien eingegangen werden, wobei es üblich ist, nur ein System (zusätzlich zur Körperakupunktur) zu verwenden und nur aktive (d.h. in der Untersuchung, z. B. durch Palpitation oder Very-Point-Technik $^{\circledR}$ nach Gleditsch, auffällige) Punkte zu behandeln.

\section{Ohrakupunktur}

In der Ohrakupunktur werden zunächst Kniepunkte, meist der ipsilateralen Seite, untersucht und ggf. genadelt. Es gibt 2 Punkte, den Punkt der chinesischen Schule (49a) und den französischen Punkt nach Nogier (49b). Als Analgesiepunkte kommen Shen Men (OP 55), Thalamus (OP 26a) und Point de Jérôme (OP 29b) in Betracht

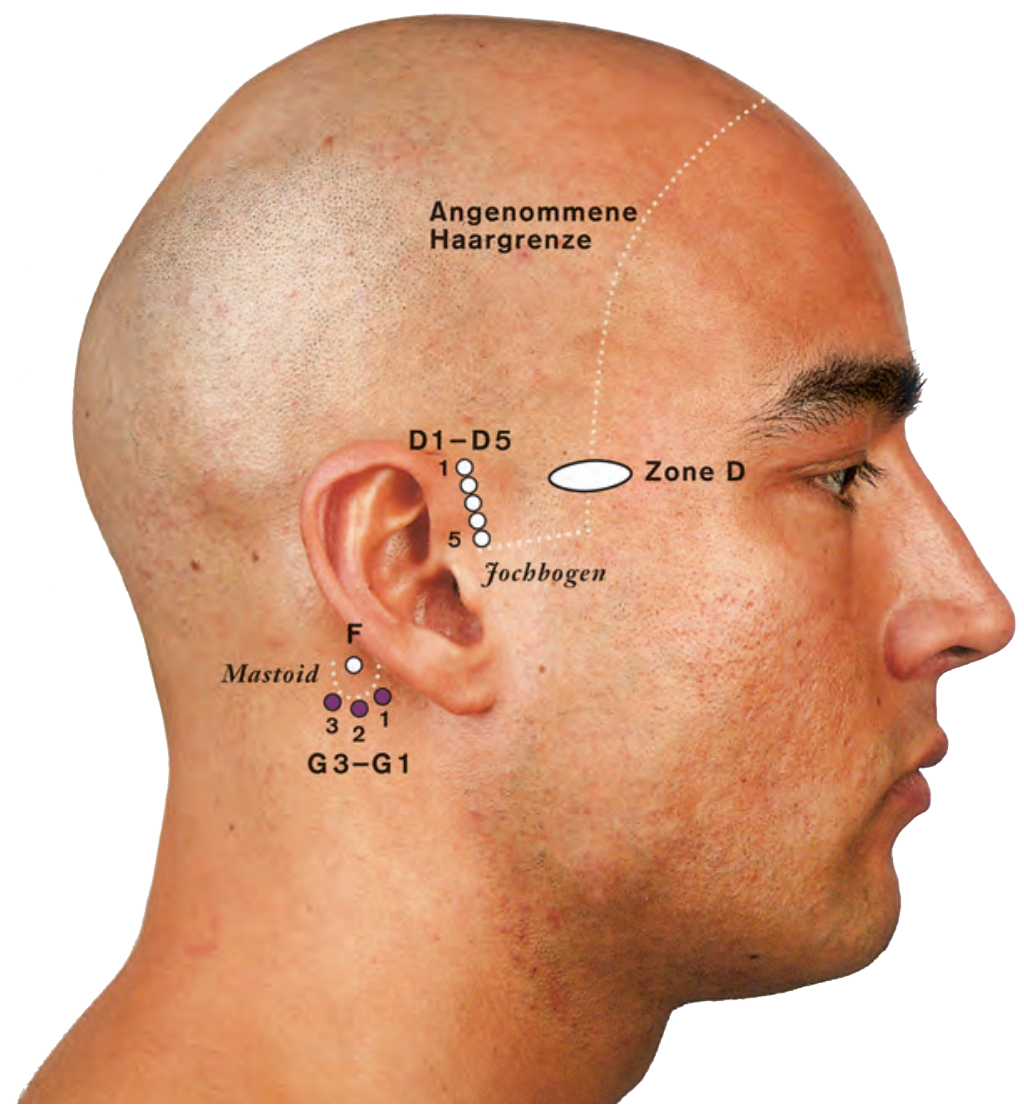

Abb. 4 YNSA-Punkte G1, G2 und G3 am Mastoid für Beschwerden im Kniebereich. @ Hecker H-U, Steveling A, Peuker ET, Hrsg. Lehrbuch und Repetitorium Ohr-, Schädel, Mund-, Handakupunktur. 4. Aufl. Stuttgart: Haug; 2010

(Abb.3). Je nach weiteren Auffälligkeiten können zusätzliche Punkte ergänzt werden wie z.B. ACTH (OP13), Graue Substanz (OP34) oder psychotrope Punkte (PT1-4).

\section{Schädelakupunktur nach Yamamoto}

Hier ist insbesondere die G-Zone um den ipsilateralen kaudalen Mastoidrand zu palpieren und bei Auffälligkeit zu behandeln. G1 am medialen/ohrnahen Rand für mediale Beschwerden, der Rand im Bereich der Mastoidspitze für dorsale und der laterale/ohrferne Rand für laterale Kniebeschwerden (Abb.4).

\section{Mundakupunktur nach Gleditsch}

Das Knie bildet sich ebenso wie die Hüfte enoral und extraoral im Bereich des Vestibulums der unteren Eckzähne ab. Enoral kann mithilfe eines Kugelstopfers das entsprechend schmerzhafte Areal palpiert und eine kleine Lokalanästhetikaquaddel gesetzt werden (vgl. dazu [11]), extraoral kann dicht am Lippensaum (nicht in der Lippe!) gegenüber des unteren Eckzahnes bzw. des enoralen Punktes mithilfe der Very-PointTechnik $^{\circledR}$ nach schmerzhaften/aktiven Punkten gesucht und behandelt werden.

Bei der Behandlung der MAPS-Punkte ist ein umgehendes Feedback des Patienten zur Verifizierung der Wirkung möglich und sinnvoll.

\section{Praktisches Vorgehen}

Das geschilderte Vorgehen setzt eine konventionelle Diagnostik sowie grundlegende Kenntnisse und Fertigkeiten des Behandlers voraus.

Um ein geeignetes Konzept für die Akupunkturbehandlung zu erstellen, ist primär Schmerzlokalisation, Ausstrahlung, sowie lokale Begleitsymptomatik zu eruieren. Die weitere anamnestische Klärung von Ursache, Auslöser, Dauer, Modalitäten, zusätzlichen körperlichen bzw. vegetativen Symptomen und psychische Belastungen machen bei Bedarf eine differenziertere TCM-Behandlung möglich. Auch Zungen- und Puls- 
diagnostik können hier weitere Hinweise liefern. Eine körperliche Untersuchung, die den gesamten Bewegungsapparat miteinbezieht, sollte bereits im Vorfeld der medizinischen Abklärung erfolgt sein. Auffälligkeiten / Beschwerden / Fehlstellungen v.a. im LWS-, Hüft- und Fußbereich können dann ggf. in die Akupunkturbehandlung mit einbezogen werden. Muskuläre Triggerpunkte sollten vor der Behandlung nochmals identifiziert werden.

Beim Behandlungsablauf empfiehlt sich (nach initialer Aufklärung über Wirkung und mögliche Nebenwirkung der Akupunktur) zunächst die Testung und ggf. Nadlung eines auffälligen MAPS-Systems mit umgehendem Feedback des Patienten bezüglich der Wirkung auf Schmerz, Bewegung oder Druckschmerz. Daran kann sich die Behandlung von lokoregionalen (Ausnahmen s.o.) und Fernpunkten anschließen. In diesem Rahmen können auch mTrP aufgesucht und behandelt werden. Eine Therapie gemäß des TCM-Syndroms kann in der Auswahl der Punkte stets mit bedacht werden, da sich hierdurch sowohl begleitende Faktoren als auch die grundlegende Konstitution beeinflussen lassen, bei komplexen und chronischen Fällen ist sie wesentlich.

In der Regel werden die Nadeln 2030 min belassen. Eine Behandlungsserie besteht aus 10-15 Sitzungen, bei chronischen Beschwerden 1-2×/Woche, bei akuten Beschwerden ggf. bis zu jedem (zweiten) Tag. Die Behandlungsfrequenz kann im Verlauf der Serie angepasst werden, z. B. initial jeden 2.-4. Tag, dann $1 \times$ /Woche, zur Stabilisierung alle 1-4 Wochen [vgl. 6, 7]. Zu beachten sind dabei ggf. die Vorgaben im Rahmen der kassenärztlichen Leistungserbringung. Auf ausreichend Ruhe, Entspannung und Wohlbefinden des Patienten sollte geachtet werden.

Abhängig von Ursache und Ausmaß der Beschwerden bei Gonarthrose ist es sinnvoll, die Akupunktur in ein Gesamtkonzept einzubetten, das weitere Therapieoptionen wie Physiotherapie, Schmerzmedikation, TENS, physikalische Maßnahmen, naturheilkundliche Anwendungen der westlichen Medizin etc. umfasst. Auch weitere Therapiesäulen der TCM wie Qi-Gong, Tuina, chinesische Arzneimitteltherapie und Diätetik können evtl. mit einbezogen werden. Insbesondere bei chronischen Schmerzen ist ein multimodaler Therapieansatz essenziell.

\section{Fallbeispiel}

Hierzu der Fall einer 73-jährigen Patientin, die wegen LWS-Syndrom und Knieschmerzen bei Gonarthrose im Rahmen des multimodalen naturheilkundlichen Therapieprogramms für chronische Schmerzen an der LMU München behandelt wurde [12]. Neben den lokoregionalen Punkten Ma 34, 36, Mi 9, 10 und Gb 34 wurden Fernpunkte wie Mi 6 und Ni 6 hinzugenommen. Ma 31 wurde gelegentlich mit einbezogen, einmalig auch getriggert. Weitere Punkte wurden aufgrund der Gesamtsymptomatik ergänzt. Die Behandlung erfolgte 4 Wochen lang $2 \times 1$ Woche zusammen mit anderen Verfahren der teilstationären Behandlung (u.a. QiGong, Atemtherapie, Meditation, Patientenseminare). Darunter besserte sich die Intensität der Knieschmerzen und konsekutiv das Gangbild. Im weiteren Verlauf kam es immer wieder zu Aggravationen, die u.a. mit Akupunkturbehandlungen begleitend behandelt wurden. Insgesamt hat sich über 2 Jahre das Schmerzniveau verbessert und die Mobilität der Patientin konnte erhalten bleiben. Auf einen Kniegelenksersatz konnte bisher verzichtet werden.

Zusammenfassend ist die Akupunkturbehandlung bei Gonarthrose eine hinreichend belegte Behandlungsmethode, die nebenwirkungsarm ist und neben anderen Behandlungsmethoden angewendet und je nach Erfahrung und Ausbildungsstand in der TCM konzeptuell erweitert werden kann.

Interessenkonflikt: Die Autorin erhält Aufwandsentschädigungen für Vorträge und Unterricht von Universität und gemeinnützigem Fachverband.

Online zu finden unter

http://dx.doi.org/10.1055/s-0036-1584365

\footnotetext{
Literatur

$\overline{1}$ Fuchs J, Rabenberg M, Scheidt-Nave C. Prävalenz ausgewählter muskuloskelettaler Erkrankungen. Ergebnisse der Studie zur Gesundheit Erwachsener in Deutschland (DEGS1). Bundesgesundheitsbl 2013; 56 (5-6): 678-686

$\overline{2}$ Tramer MR, Moore RA, Reynolds DJ, McQuay HJ. Quantitative estimation of rare adverse events which follow a biological progression: a new model applied to chronic NSAID use. Pain 2000; 85: 169-182

$\overline{3}$ Vickers A, Cronin A, Maschino A et al. Acupuncture for chronic pain: individual patient data metaanalysis. Arch Intern Med 2012; 127 (19): 1444-1453
}

4 Witt C, Brinkhaus B, Jena S et al. Acupuncture in patients with osteoarthritis of the knee: a randomised trial. Lancet 2005; 366 (9480): 136143

5 Scharf HP, Mansmann U, Streitberger K, Witte S, Krämer J, Maier C, Trampisch HJ, Victor N. Acupunctur and knee osteoarthritis: a three-armed randomized trial. Ann of Int Medicine 2006; 145 (1): 12-20

6 Focks C, Hillenbrand N, Hrsg. Leitfaden Traditionelle chinesische Medizin. München: Urban\&Fischer, Elsevier; 2001

7 Bachmann J. Chinesische Medizin in der Orthopädie. München: Urban\&Fischer, Elsevier; 2008

8 Hempen C. dtv Atlas Akupunktur. München: Deutscher Taschenbuch Verlag; 1997

9 König G, Wancura I. Praxis und Theorie der Neuen Chinesischen Akupunktur. Wien: Verlag Wilhelm Maudrich; 1989

$\overline{10}$ Irnich D. Leitfaden Triggerpunktbehandlung. München: Urban\&Fischer, Elsevier; 2009

$\overline{11}$ Gleditsch J. Mikro-Aku-Punkt-Systeme. Grundlagen und Praxis der somatotopischen Therapie. Stuttgart: Hippokrates; 2002

$\overline{12}$ Irnich D. Das Münchner Naturheilkundliche Schmerzintensivprogramm - Rücken. ZKM 2014; 6 (2): 12-19

\section{Dr. med. Franziska Portzky}

Interdisziplinäre Schmerzambulanz

Klinikum der Universität München, Innenstadt

Pettenkoferstr. 8a

80366 München

franziska.portzky@web.de

Franziska Portzky ist Fachärztin für Allgemeinmedizin mit Zusatzbezeichnung Akupunktur und Naturheilverfahren. Sie ist Teil des Akupunkturteams, das Patienten des multimodalen Therapieprogramms für chronische Schmerzen der LMU München, Klinikum Innenstadt behandelt. Daneben besteht Lehrtätigkeit für Akupunktur bei der DÄGfA. 